\title{
Die Zürcher Arzt-Apotheker-Familie Lavater und Johann Wolfgang von Goethe
}

Antje Mannetstätter und Christoph Friedrich

\section{Summary}

The aim of this paper is to demonstrate the connections between Johann Wolfgang von Goethe (1749-1832) and the physician and pharmacist family Lavater in Zurich. The analysis of the correspondence between Johann Bartholomäus Trommsdorff (1770-1837) and the Lavater family and between the Lavater family and the government of the duchy of Weimar shows an interesting story about a picture "Goethe in Italy".

\section{Zusammenfassung}

Anliegen des Aufsatzes ist es, die Beziehung zwischen Johann Wolfgang von Goethe (1749-1832) und der Familie Lavater, die Ärzte und Apotheker in Zürich waren, aufzuzeigen. Die Analyse der Korrespondenz zwischen Johann Bartholomäus Trommsdorff (1770-1837) und der Familie Lavater sowie der Familie Lavater mit der Regierung des Grossherzogtums Weimar widerspiegelt die interessante Geschichte des Bildes «Goethe in Italien».

In einem für die Edition des Briefwechsels Johann Bartholomäus Trommsdorffs (1770-1837) transkribierten Brief des Zürcher Arztes und Apothekers Diethelm Heinrich Lavater II (1781-1846) fand sich die folgende Bemerkung: «Ich besitze ein in länglichem Octav wohl scizzirtes [!] Bild in liegender Stellung von Göthe ...»1

1 Nachlass 259 J. B. Trommsdorff und Nachf. Brief von Diethelm Lavater II an Johann Bartholomäus Trommsdorff vom 23. April 1832, Staatsbibliothek zu Berlin, Preussischer Kulturbesitz, Handschriftenabteilung.

Apothekerin Antje Mannetstätter, Prof. Dr. rer. nat. Dipl.-Hist. Christoph Friedrich, Institut für Pharmazie, Lehrstuhl Geschichte der Pharmazie, Friedrich-Ludwig-Jahn-Str. 17, D-17487 Greifswald 


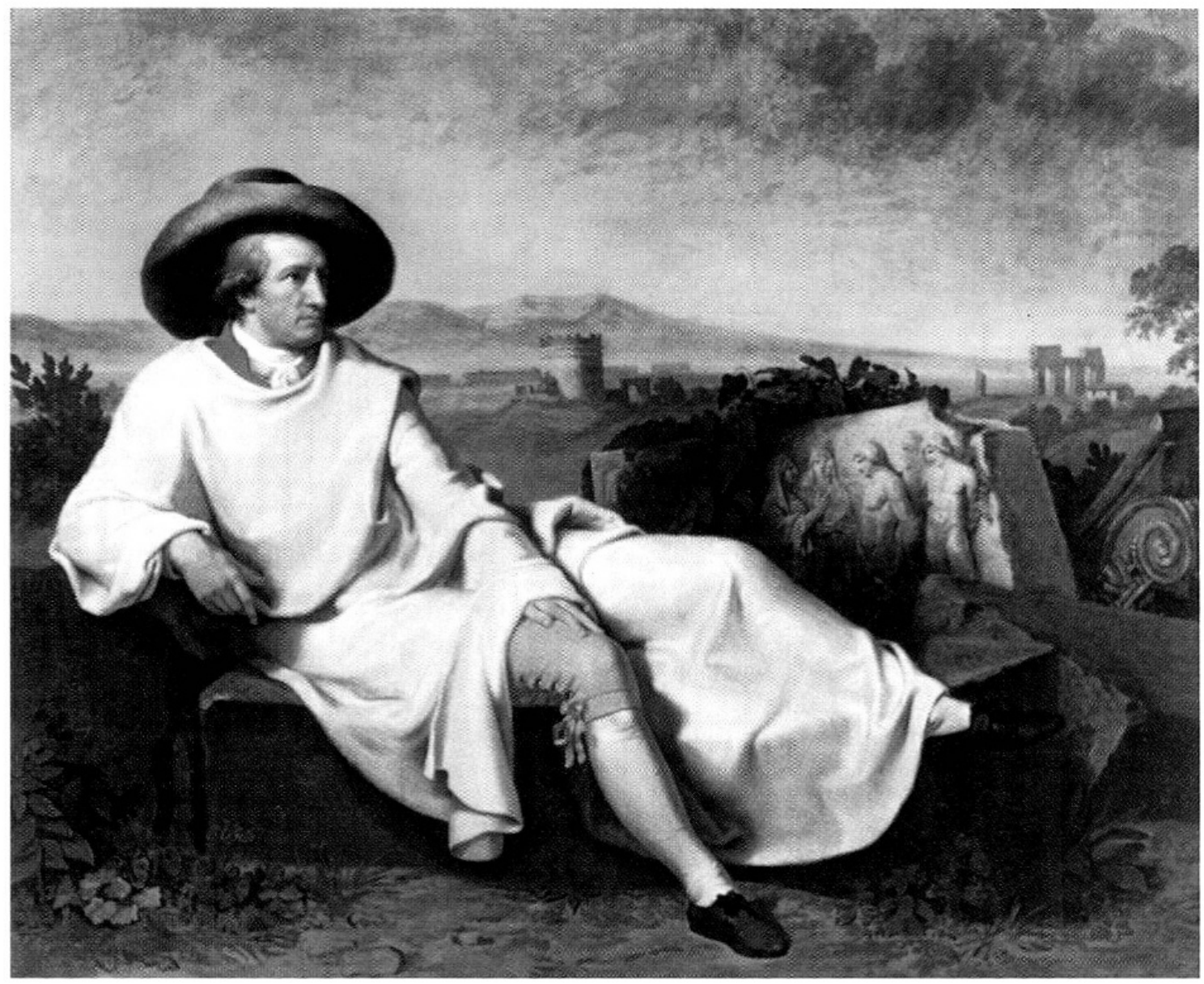

Abb. 1. J.H.W. Tischbein «Goethe in der Campagna di Roma», Städelsches Kunstinstitut Frankfurt am Main.

Diese lapidare Aussage gab den Anlass zu weiteren Nachforschungen über Aussehen, Entstehung und Verbleib dieser Zeichnung. Im Archiv der Familie Lavater in Zürich fanden sich noch weitere Briefe mit Vertretern des Weimarer Hofes, die über diese pharmazie- und kunsthistorisch interessante Begebenheit Aufschluss gaben ${ }^{2}$. Die Zeichnung, welche heute im GoetheNationalmuseum in Weimar verwahrt wird, ähnelt dem Ölgemälde «Goethe in der Campagna di Roma» von Johann Heinrich Wilhelm Tischbein (1751-1829) - dem Sinnbild für Goethes italienische Reise, das sich heute im

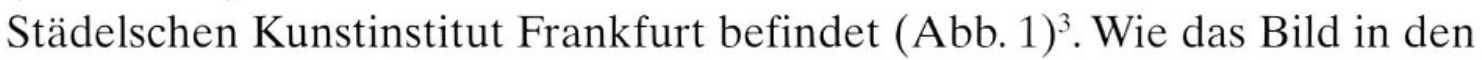
Besitz der Familie Lavater gelangte und welche Beziehungen zu Johann Wolfgang von Goethe (1749-1832), aber auch zu Tischbein bestanden, soll nachfolgend näher beleuchtet werden.

2 Zentralbibliothek Zürich, FA Lavater 1781/8, 12.

3 K. Muthesius, Die Schicksale eines Goethebildes. Jahrbuch der Goethe-Gesellschaft 12 (1926) 229-238. 


\section{Goethe und die Pharmazie}

Während eines krankheitsbedingten Aufenthaltes in seinem Frankfurter Elternhaus beschäftigte sich Goethe 1768 mit Alchemie und Mystik. In seinem Giebelzimmer richtete er ein kleines chemisches Laboratorium ein. Nachdem er im April 1770 zu Studienzwecken in Strassburg eingetroffen war, besuchte er neben juristischen auch naturwissenschaftliche Vorlesungen ${ }^{4}$. Sein erster akademischer Lehrer auf dem Gebiet der Chemie wurde der Apotheker und Arzt Jakob Reinbold Spielmann (1722-1783), der seinen theoretischen Unterricht mit praktischen Experimenten im Laboratorium der Hirsch-Apotheke Strassburg ergänzte ${ }^{5}$.Während dieser Zeit und nach seiner Übersiedlung nach Weimar 1775 vollzog sich ein Wandel in Goethes Auffassung über die Pharmazie. Betrachtete er in seinen Jugendjahren und zu Beginn der Strassburger Zeit diese Wissenschaft eher spöttisch, faszinierten ihn später das Systematische und die Beschreibung chemischer Phänomene, denn die Apotheker erwarben ihre wissenschaftlichen Erkenntnisse weitestgehend auf experimenteller Basis ${ }^{6}$. In Weimar und Jena übernahm Goethe die Oberaufsicht über die naturwissenschaftlichen Anstalten, wie den Botanischen Garten, die Tierarzneischule, das mineralische, botanische und physikalisch-chemische Kabinett. Er beschäftigte sich mit dem Bergbau und somit auch mit Mineralogie. Zu seinem wissenschaftlichen Berater wählte er wiederum einen Apotheker - Wilhelm Heinrich Sebastian Bucholz (1743-1798)-, den Besitzer der Hofapotheke Weimar, Bergrat und Hausarzt am Grossherzoglichen Hofe. Goethe war fasziniert von dessen breiten wissenschaftlichen Kenntnissen ${ }^{7}$. Angeregt durch den Aufschwung der Chemie in der zweiten Hälfte des 18. Jahrhunderts bemühte sich Goethe um die Schaffung eines separaten Lehrstuhls für Chemie an der Universität Jena. Er schlug für diese Professur einen Schüler von Bucholz, Johann Friedrich August Göttling (1753-1809), vor. Diesem wurde auf Goethes Empfehlung durch den Grossherzog Carl August ein zweijähriger Studienaufenthalt in Göttingen sowie eine Reise nach England und Holland ermöglicht. 1789 begann Göttling seine Lehrtätigkeit an der Universität Jena. In Fragen der Physik, Chemie und Mineralogie erwies er sich als zuverlässiger Berater

4 O. Zekert, Deutsche Apotheker, Berlin/Wien, 1942, 52.

5 U. Grass, Zu Leben und Werk von Jakob Reinbold Spielmann (1722-1783), Stuttgart, 1983.

6 G. Urdang, Goethe und die Pharmazie. Dtsch. Apoth.-Ztg. 94 (1954) 209-212.

$7 \mathrm{Vgl}$. (4), 55-56. Bucholz wurde in Bernburg geboren und erlernte in Magdeburg die Apothekerkunst. 1761 studierte er in Jena Medizin und wurde dort 1764 zum Dr. med. promoviert. Anschliessend betrieb er eine Praxis in Weimar und wurde 1777 zum Hofmedikus ernannt. Zugleich leitete er die dortige Hofapotheke. Vgl. Deutsche ApothekerBiographie, Bd. 1, Stuttgart, 1975, 91f. 
Goethes und wurde von ihm auch weiterhin finanziell bei der Errichtung eines Laboratoriums unterstützt ${ }^{8}$. Als der Lehrstuhl 1809 nach Göttlings Tod erneut zur Vergabe stand, schlug Goethe mit Johann Bartholomäus Trommsdorff einen weiteren Bucholz-Schüler vor, riet jedoch abzuwarten. Der vom Herzog Carl August von Sachsen-Weimar (1757-1828) um Rat befragte Münchner Professor Adolf Ferdinand Gehlen (1775-1815) $)^{9}$ empfahl den arbeitslosen Pharmaziegehilfen Johann Wolfgang Döbereiner $(1780-1849)^{10}$. Trotz Bedenken der Philosophischen Fakultät der Universität Jena wurde Döbereiner 1810 Nachfolger Göttlings. Seine aussergewöhnlichen Fähigkeiten rechtfertigten in der Folgezeit diese Entscheidung und überzeugten auch Goethe, dessen anfängliche Reserviertheit sich in Achtung und später sogar Freundschaft wandelte. Dies belegt die umfangreiche Korrespondenz von Goethe und Döbereiner sowie die Tatsache, dass letzterer spätere Berufungen an grössere Universitäten stets mit der Begründung der Treue zum Hof und zu Goethe ablehnte. Zudem unterstützte Goethe auch finanziell bei der Anschaffung von Glasapparaturen und Laborgeräten aus Platin ${ }^{11}$. Spielmann, Bucholz, Göttling und Döbereiner sind wohl diejenigen Apotheker, die den grössten Einfluss auf Goethes naturwissenschaftliche Betätigung nahmen, doch es gibt noch eine Reihe weiterer Phar-

8 Wie Anm. 6 sowie W. Aigner, Die Beiträge des Apothekers Johann Friedrich Göttling (1755-1809) zur Entwicklung der Pharmazie und Sauerstoffchemie, Dissertation Universität München 1985 [hier falsches Geburtsdatum] und Ch. Friedrich, J. F. A, Göttling und die Pharmazie, in: Haeckeliana. Abhandlungen zur Wissenschaftsgeschichte, H. 2 (1993) 63-72. Göttling, der in Derrenburg geboren wurde, begann 1769 seine Apothekerlehre bei Johann Christian Wiegleb (1732-1800) in Langensalza. 1774 trat er eine Stelle in der Weimarer HofApotheke bei Bucholz an.

9 Gehlen studierte Pharmazie bei Karl Gottfried Hagen (1749-1829) in Königsberg und wurde dort zum Dr. med. promoviert. Seine Ausbildung setzte er in Berlin bei Martin Heinrich Klaproth (1743-1817) und in Halle bei dem Mediziner Johann Christian Reil (1759-1813) fort, wo er sich habilitierte. 1807 übernahm er den Aufbau des Chemischen Laboratoriums in München. Er verstarb 1815 an einer Arsenwasserstoffvergiftung. Kurz vor seinem Tode begründete er die Zeitschrift «Repertorium für die Pharmacie», die Johann Andreas Buchner (1783-1852) fortsetzte. Vgl. Deutsche Apotheker-Biographie, Bd. 1, Stuttgart, 1975, 194.

10 Der auf dem Rittergut Burg bei Hof in ärmlichen Verhältnissen aufgewachsene Döbereiner begann 1794 seine pharmazeutische Ausbildung in der Apotheke zu Münchberg. Als Gehilfe wirkte er nach 1797 in Dillenburg, Karlsruhe und Strassburg. 1802 eröffnete er in Gefrees eine Drogen- und Landesproduktehandlung nebst einer kleinen chemischen Fabrik, die er jedoch 1806 wieder schliessen musste. Er arbeitete anschliessend in der Textilmanufaktur seines Schwagers in Münchberg und 1808 schliesslich als Verwalter des Gutes St. Johannis bei Bayreuth. Hier erreichte Döbereiner, der die ganzen Jahre wissenschaftlich gearbeitet hatte, der Ruf nach Jena, wo er als einer der erfolgreichsten Lehrer der Chemie und Pharmazie wirkte. Vgl. Deutsche Apotheker-Biographie, Bd. 1, Stuttgart, 1975, 123-126.

11 Wie Anm. 6 sowie O. Zekert, Goethe als Förderer der Naturwissenschaften, Wien, 1953, 23-33 und A. Adlung und G. Urdang, Grundriss der Geschichte der deutschen Pharmazie, Berlin, 1935, 454. 
mazeuten, die zu seinem Bekanntenkreis zählten, so z. B. Friedlieb Ferdinand Runge (1794-1867) ${ }^{12}$, Christian Gottlob Gmelin (1749-1809) ${ }^{13}$, Karl Ludwig Fernow (1763-1808) ${ }^{14}$ und Emmanuel Christian Wilhelmi (1745-1827) ${ }^{15}$. So wurden auch Goethes meteorologische Studien massgeblich durch Apotheker beeinflusst, namentlich durch den Engländer Luke Howard und Rudolph Brandes (1795-1842). Brandes veranlasste 1822 die Ernennung des Dichters zum Ehrenmitglied des «Apothekervereins im Nördlichen Teutschland» ${ }^{16}$.

Beziehungen bestanden indessen nicht nur zu deutschen Apothekern, sondern auch zu schweizerischen, so insbesondere zu der Arzt-ApothekerFamilie Lavater.

\section{Die Verbindung zwischen Goethe und der Familie Lavater}

Der wohl bekannteste Vertreter der Familie Lavater, der Theologe und Philosoph Johann Kaspar Lavater (1741-1801), wurde als 14. Kind des Apothekerarztes Johann Heinrich Lavater (1698-1774) in Zürich geboren ${ }^{17}$.

12 Runge begann 1810 seine Apothekerausbildung in der Rats-Apotheke zu Lübeck. Nach 1816 studierte er Medizin in Berlin und Göttingen. 1818 setzte er das Studium der Chemie in Jena fort und wurde hier zum Dr. phil. promoviert. Er habilitierte sich an der Universität Berlin und las hier über Tier- und Pflanzenchemie. In Breslau avancierte er zum Professor für Technologie. Seit Anfang der 30er Jahre leitete er eine Fabrik in Oranienburg. Runge war der Entdecker des Anilins, Phenols und Koffeins und der mydriatischen Wirkung von Atropa belladonna, die er auch Goethe vorführte.Vgl. B. Anft, Fr. F. Runge sein Leben und sein Werk, Berlin, 1937.

13 Christian Gottlob Gmelin entstammte einer Tübinger Apothekerfamilie und begann in seiner Heimatstadt seine pharmazeutische Ausbildung. Er wirkte als Gehilfe in Schaffhausen, Karlsruhe, Frankfurt/M. und Hannover, ehe er die Tübinger Apotheke übernahm. Er studierte ausserdem noch Medizin und promovierte 1785 an der Universität Erlangen. Vgl. Deutsche Apotheker-Biographie, Bd. 1, Stuttgart, 1975, 209.

14 Fernow, der in Blumenhagen in der Uckermarck geboren wurde, begann 1777 seine pharmazeutische Ausbildung in Anklam und wirkte anschliessend in Apotheken in Altona, Hamburg und Lübeck. 1788 wandte er sich der Malerei zu und studierte von 1791 bis 1793 in Jena Philosophie. Anschliessend ging er nach Rom und wurde 1802 als ausserordentlicher Professor der Philosophie nach Jena berufen. Er trat vor allem als Kunsthistoriker hervor. Seine Kontakte zu Goethe betrafen somit weniger pharmazeutisch-chemische Fragen. Vgl. Deutsche Apotheker-Biographie, Bd. 1, Stuttgart, 1975, 159f.

15 Als Sohn eines Buchbinders erlernte Wilhelmi die Pharmazie in der Hof-Apotheke Jena, die er 1767 übernehmen konnte. Seine Kontakte zu Goethe werden als eher «frostig» geschildert. Vgl. Deutsche Apotheker-Biographie, Bd. 2, Stuttgart, 1978, 750.

16 Wie Anm. 6. Brandes, der in Salzuflen geboren wurde, begann seine pharmazeutische Ausbildung in Osnabrück. Er studierte in Halle Medizin und wurde dort 1817 zum Dr. med. promoviert. 1817 setzte er seine Ausbildung bei Bucholz in Weimar fort und übernahm 1819 die väterliche Apotheke in Salzuflen. Er gehörte zu den Begründern des «Apothekervereins im Nördlichen Teutschland». Vgl. H. Zimmermann, Simon Rudolph Brandes (1795-1842). Ein bedeutender Apotheker des 19. Jahrhunderts, Stuttgart, 1985.

17 Hans Conrad Lavater (1628-1691) begründete die 235jährige Apothekertradition der Familie Lavater in Zürich, vgl. dazu F. Ledermann (Hrsg.), Schweizer Apotheker-Biographie, Bern, 1993, 210-214. 
Berühmtheit erlangte Johann Kaspar durch seine Physiognomie-Studien, an denen auch Goethe mehrere Jahre mitwirkte. Lavater war der Überzeugung, dass sich das seelische Leben eines Menschen in seinen Gesichtszügen und Körperformen widerspiegele. Für sein Hauptwerk, die «Physiognomischen Fragmente zur Beförderung der Menschenkenntnis und der Menschenliebe», wurde ihm nicht nur durch Goethe, sondern auch den Weimarer und Anhaltinischen Hof grösste Anerkennung zuteil ${ }^{18}$. Während seiner Schweizer Reisen besuchte Goethe Lavater mehrmals in Zürich, und eine Mitteilung an Charlotte von Stein (1742-1827) aus dem Jahre 1779 spiegelt sein Verhältnis zu diesem wider: «Die Bekanntschaft von Lavatern ist für den Herzog und mich, was ich gehofft habe, Siegel und oberste Spitze der ganzen Reise und eine Weide an Himmelsbrot, wovon man lange gute Folgen spüren wird ...» ${ }^{19}$

Diethelm Lavater I (1743-1826), der Bruder von Johann Kaspar, war wie sein Vater in der Doppelfunktion als Arzt und Apotheker tätig. Erste Kontakte zu Goethe knüpfte er bereits während seiner Studienzeit, denn in der Tischrunde des Leipziger Professors Christian Gottlieb Ludwig (1709-1773), bei dem er wohnte, war der junge Goethe häufig zu Gast ${ }^{20}$. Obwohl sich aus diesen Begegnungen keine dauerhafte Freundschaft entwickelte, besuchte Goethe Diethelm Lavater I 1797 in Zürich ${ }^{21}$. Der Grund dieses Treffens bleibt verborgen, Goethes Besuch dürfte aber nicht Johann Kaspar Lavater gegolten haben, da zwischen diesem und Goethe bereits 1780 eine Entfremdung eingetreten war. Durch Diethelm Lavater I erhielt Goethe einen Einblick in die Freimaurerei, denn Lavater war massgebendes Gründungsmitglied der Loge «Modestia cum Libertate», deren Treffen Goethe bei seiner Schweizer Reise 1775 besuchte und die den Anstoss zu seinem Beitritt in die Loge «Amalia» in Weimar 1779 gegeben haben sollen ${ }^{22}$.

Durch Vermittlung Johann Kaspar Lavaters lernte Goethe auch den Maler Johann Heinrich Wilhelm Tischbein (1751-1829) kennen. Tischbein, der einer Künstlerfamilie entstammte, studierte nach einer Lehre bei seinem Onkel in Kassel und Aufenthalten in Hamburg, Berlin und Holland ab 1779 an der Akademie des Schweizer Bildhauers Alexander Trippel (1744-1793) in Rom. Das Ablaufen seines Stipendiums veranlasste ihn, Italien zu verlassen, um nach Frankreich zu gehen. Völlig mittellos in der Schweiz angelangt,

18 O. Krätz, Goethe und die Naturwissenschaften, München, 1992, 32-43.

19 Vgl. (18), 64

20 O. Zuber, Br. Diethelm Lavater. Sonderdruck aus: Quatuor Coronati Jahrbuch, Hamburg, 1980,137-159.

21 J. Kruse, Johann Heinrich Lips 1758-1817. Ein Zürcher Kupferstecher zwischen Lavater und Goethe, Coburg, 1989, 55.

22 Vgl. dazu Anm. 20. 
fand er im Hause Johann Kaspar Lavaters freundliche Aufnahme. Dieser nutzte Tischbeins Kenntnisse in der Portraitmalerei für seine PhysiognomieStudien. Nachdem Lavater ihm in Zürich einige Aufträge verschafft hatte, sandte er Werke Tischbeins nebst Empfehlungen nach Weimar, wodurch auch Goethe auf den Maler aufmerksam wurde ${ }^{23}$. Er empfahl ihn Herzog Ernst II. von Gotha (1745-1804), der Tischbein anstellte, jedoch einen erneuten Studienaufenthalt in Italien forderte. Von dort aus hielt Tischbein ständigen Kontakt zu Goethe, der bei seiner Ankunft in Rom 1786 in Tischbeins Wohnung zog. Während dieser Zeit entstand das Gemälde «Goethe in der Campagna di Roma», das später zum Sinnbild für Goethes italienische Reise werden sollte ${ }^{24}$. Wie im Falle Johann Kaspar Lavaters distanzierte sich Goethe in späteren Jahren auch von Tischbein, da er sich von diesem ausgenutzt fühlte. Trotz Bemühungen von beiden Seiten erreichte ihr Verhältnis nie wieder die Intensität der römischen Zeit ${ }^{25}$.

Während seines Aufenthaltes in Zürich lernte Tischbein, möglicherweise durch Vermittlung Johann Kaspar Lavaters, Diethelm Lavater I kennen, der Tischbein während seiner Studienzeit 1799-1800 in Göttingen besuchte. Diese Bekanntschaft wurde auch auf Diethelm Lavater II (1781-1846), der wie sein Vater Diethelm I Arzt und Apotheker war, übertragen ${ }^{26}$. Als Kunstsammler seinem Vater folgend, übernahm Diethelm II aus dessen Erbteil eine Reihe Bilder des Malers. Möglicherweise waren die Gemälde durch Schenkung Tischbeins in den Besitz der Familie Lavater gelangt, es ist indessen auch erwiesen, dass Johann Kaspar Lavater durch den Ankauf von Portraits häufig die finanziellen Mittel der Familie überbeanspruchte ${ }^{27}$. Vielleicht wurde der Geldmangel durch Verkauf der Bilder innerhalb der Familie ausgeglichen, denn Diethelm I war ebenfalls passionierter Kunstsammler, jedoch durch die Apotheke und Arztpraxis finanziell abgesichert ${ }^{28}$.

\section{Ein schweizerisch-deutscher Kunsttransfer}

Diethelm Lavater II hatte seine pharmazeutische Ausbildung von 1796 bis 1798 am Chemisch-physikalisch-pharmaceutischen Institut Johann Bartho-

23 Ch. Lenz, Tischbein. Goethe in der Campagna di Roma, Druck des Städelschen Kunstinstitutes, Frankfurt am Main, 1979, 23-26.

24 G. Schwedt, Goethe. Museen, Orte, Reiserouten, München, 1996, 143.

25 Wie Anm. 23.

26 Entwurf eines Briefes von Diethelm Lavater II an den Kanzler v. Müller vom 29. Mai 1832, FA Lavater 1781/12, Zentralbibliothek Zürich.

27 M. Bircher und G. Lammel, Helvetien in Deutschland. Schweizer Kunst aus Residenzen deutscher Klassik 1770-1830, Zürich, 1990/91, 108.

28 Wie Anm. 3. 


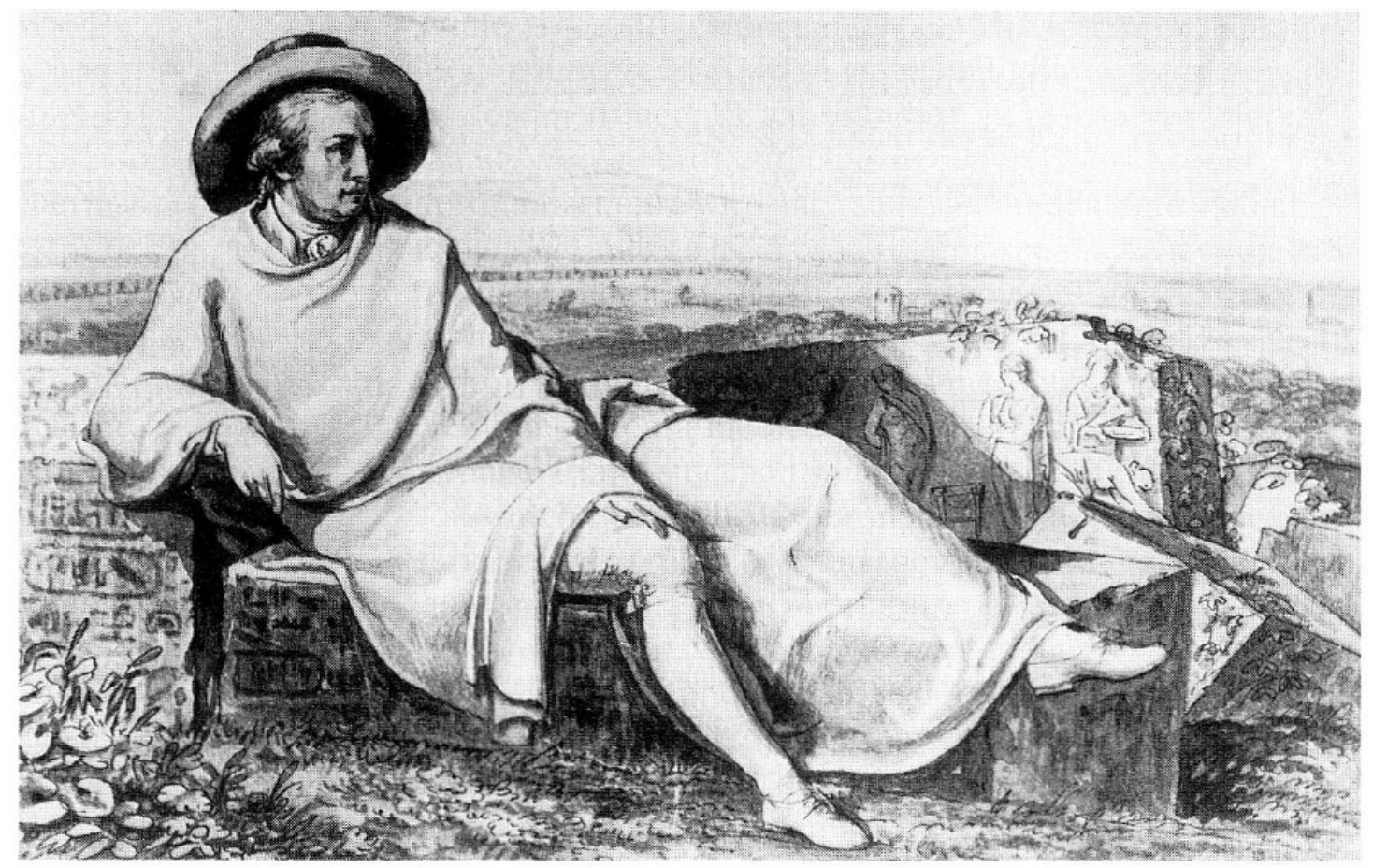

Abb. 2. J.H.W. Tischbein, J.G. Schütz, F. Büry «Goethe in der Campagna di Roma», GoetheNationalmuseum Weimar.

lomäus Trommsdorffs in Erfurt absolviert. Trommsdorff, Sohn eines Erfurter Medizinprofessors und Apothekenbesitzers, hatte 1784 seine Apothekenlehrzeit in der Hof-Apotheke Weimar begonnen. 1790 übernahm er die Erfurter Schwan-Ring-Apotheke, die er bis zu seinem Tode leitete. An der Universität Erfurt wurde er 1794 zum Dr. phil. promoviert und avancierte 1811 zum ordentlichen Professor der Medizinischen Fakultät. 1795 eröffnete Trommsdorff sein chemisch-pharmazeutisches Privatinstitut, an dem bis 1828 über 300 Personen, darunter zahlreiche Apotheker und Gewerbetreibende, ihre Ausbildung erhielten. Die dort kreierte Unterrichtsmethode erlangte auch für andere naturwissenschaftliche Studiengänge Modellcharakter. Trommsdorff gilt als Begründer einer wissenschaftlichen Pharmazie und trug dazu u.a. mit dem von ihm herausgegebenen «Journal der Pharmacie» (1793-1817, danach als «Neues Journal der Pharmacie» bis 1834), 512 Aufsätzen sowie mit 34 Lehr- und Handbüchern bei. Diethelm Lavater II darf wohl als sein bedeutendster schweizerischer Schüler betrachtet werden.

Dieser studierte 1798 Medizin in Jena und Göttingen, wo er 1800 zum Dr. med. promoviert wurde. Ab 1800 arbeitete er zunächst in der Arztpraxis seines Vaters und übernahm nach dessen Tod 1826 auch seine Apotheke ${ }^{29}$. Die enge Beziehung zu seinem Lehrer Trommsdorff hielt er lebenslang auf-

29 Wie Anm. 17, S. 212. 
recht und bezog ihn in wichtige Entscheidungen ein $^{30}$; so auch als er sich nach Goethes Tod im März 1832 an eine Zeichnung erinnerte, die er von seinem Vater geerbt hatte und die nun «in der obersten Stube» hing (Abb. 2) $)^{31}$. Da er wusste, wie sehr man am Weimarer Hof um Goethe trauerte und welchen Wert man den sogenannten «Goetheschen Reliquien ${ }^{32}$ beimass, wandte er sich mit seinen Plänen zunächst an Trommsdorff. Lavater wusste um dessen Beziehungen zum Hof und wollte sich zunächst dessen Zustimmung versichern. In einem Brief an Johann B. Trommsdorff schrieb er:

«Wie glauben Sie nun? - Dürfte ich dieselbe [Zeichnung, d. V.] etwa mit ein paar Dutzend schöner lithogr[aphischer] Abdrücke - an den Grossherzog selbst addressiren? Fürchten Sie nicht, es würde solche Übersendung schiefe Beurtheilung auf mich laden - denn um aller Welt willen möchte ich mich nicht blamiren oder auf irgend eine Weise prostituiren lassen - noch weniger gar in den Verdacht eines Schmeichlers oder Zudringlichen kommen.» ${ }^{33}$

Trommsdorff empfahl Lavater in seinem Antwortschreiben vom 1. Mai 1832, das Bild an Kanzler Friedrich von Müller (1779-1849), einen engen Vertrauten Goethes und des Weimarer Hofes, zu übersenden. Dabei versicherte er Lavater:

«Auf keinen Fall dürfen Sie eine Missdeutung befürchten, dafür sind Sie schon durch Ihre Stellung geschützt.» ${ }^{34}$

Ermutigt durch Trommsdorffs Zustimmung wandte sich Diethelm Lavater II nun an den Kanzler von Müller ${ }^{35}$. Wie schon Trommsdorffs Antwortschreiben, zeigt auch das des Kanzlers von Müller deutlich, in welchem Ansehen der Name Lavater zu dieser Zeit in Weimar stand:

«Der Name des Gebers wird in Weimar die Erinnerung an jene schönen Zeiten erneuen, wo zwischen Ihrem edlen Oheim, unserem verewigten ruhmwürdigen Fürstenpaare und Göthe ein so ausgezeichnetes Band wechselseitiger Achtung und Zuneigung geknüpft war! $»^{36}$

Erfreut über diese positive Reaktion, übersandte Lavater die Zeichnung sowie einige Lithographien am 29. Mai 1832 nach Weimar. Beigegeben waren ein Brief an Kanzler von Müller, weitere Bilder von Diethelm Lavater I,

30 W. Götz, Die Beziehung zwischen der Zürcher Apothekerfamilie Lavater und Johann Bartholomäus Trommsdorff, Erfurt, Gesnerus 43, 1986, 299-311.

31 Brief von Diethelm Lavater II an Johannes Lavater-Hirzel vom 24. April 1832, FA Lavater 1781/8, Zentralbibliothek Zürich.

32 Brief des Kanzlers v. Müller an Diethelm Lavater II vom 22. Mai 1832, FA Lavater 1781/14, Zentralbibliothek Zürich.

33 Vgl. dazu Anm. 1.

34 Brief von Johann Bartholomäus Trommsdorff an Diethelm Lavater II vom 1. Mai 1832,FA Lavater 1781/14, Zentralbibliothek Zürich.

35 F. Zollinger, Goethe in der Campagna bei Rom, Zürich, 1927, 10-11.

36 Brief des Kanzlers v. Müller an Diethelm Lavater II vom 22. Mai 1832, FA Lavater 1781/14, Zentralbibliothek Zürich. 
Johann K. Lavater sowie ein Doppelbild Johann K. Lavaters mit seinem Sohn Heinrich (1768-1819). Zudem äusserte Diethelm Lavater II den Wunsch, auch seinem «lieben Freund Trommsdorff» eine Lithographie zukommen zu lassen $^{37}$. Diethelm Lavater II hatte mit der Versendung der Lithographien sein Ziel erreicht: die «Original Zeichnung sollte, ihrer Seltenheit wegen, an den Ort gelangen, wo G[oethe] besonders gelebt und gewirkt hat .... ${ }^{38} \mathrm{Um}$ so mehr erfreuten ihn die zahlreichen Dankschreiben, die nun bei ihm eingingen. Kanzler von Müller schrieb am 23. Juni 1832:

«Euer Wohlgebohrn kann ich nicht genug schildern, wie grosse Freude Ihre geneigten Zusendungen des Goetheschen Bildes und der schönen Lithographien gemacht haben. Die Aehnlichkeit des Bildes ist so gross, dass selbst die späteren Jahre sie nicht verwischt haben und das Ganze sehr geistvoll componirt.» ${ }^{39}$

Beigegeben waren diesem Schreiben ein Dankesbrief der Grossherzogin Maria Pawlowna (1786-1859) nebst drei Medaillen sowie drei Gedichte aus der Feder von Müllers ${ }^{40}$. Später folgte noch ein persönliches Schreiben des Grossherzogs Carl Friedrich von Sachsen-Weimar (1783-1853), der ebenfalls als Geschenk eine Medaille beilegte ${ }^{41}$.

Ermutigt von dem Erfolg der Schenkung kam Diethelm Lavater II wohl die Idee, weitere Lithographien über Kunsthändler zu veräussern. Zu diesem Zweck schrieb er an seinen Sohn, Johannes Lavater-Hirzel (1812-1888), der nach seiner Apothekerlehre 1832 in Mannheim konditionierte, und bat diesen, die Lithographien dort anzubieten ${ }^{42}$. Späteren Briefen an Johannes Lavater-Hirzel konnte entnommen werden, dass die Nachfrage wohl doch nicht den Erwartungen entsprach.

\section{Entstehung der Zeichnung}

Diethelm Lavater II war bis zu seinem ersten Schreiben an Kanzler von Müller überzeugt, eine Originalzeichnung Goethes zu besitzen, wie ihm dies sein Vater, aus dessen Besitz das Bild stammte, übermittelt hatte. Demnach sollte Goethe die durch ihn selbst verfertigte Zeichnung einem seiner Freunde, dem Musiker Philipp Christoph Kayser (1755-1823), der ihn auf

$37 \mathrm{Vgl}$. Anm. 26.

38 Vgl. Anm. 1.

39 Brief des Kanzlers v. Müller an Diethelm Lavater II vom 23. Juni 1832, FA Lavater 1781/14, Zentralbibliothek Zürich.

40 Brief der Grossherzogin Maria v. Sachsen-Weimar an Diethelm Lavater II vom 18. Juni 1832, FA Lavater 1781/14, Zentralbibliothek Zürich.

41 Brief des Grossherzoges Carl v. Sachsen-Weimar an Diethelm Lavater II vom 12. August 1832, FA Lavater 1781/14, Zentralbibliothek Zürich.

42 Briefe von Diethelm Lavater II an Johannes Lavater-Hirzel vom 21. Juni und 12. Juli 1832, FA Lavater 1781/8, Zentralbibliothek Zürich. 
einer seiner Reisen nach Italien begleitet hatte, geschenkt haben. Kayser, der sich später in Zürich niederliess, wurde von Diethelm Lavater I medizinisch betreut, und dieser erwarb das Goethe-Bild nach Kaysers Tod 1823 aus dessen Nachlass. Zuvor hatte Kayser ihm noch versichert, dass es sich um ein Original Goethes handelte. Die so von Diethelm Lavater II weitergegebene Entstehungsgeschichte der Zeichnung wurde durch ein Schreiben von Müllers vom 22. Mai 1832 erstmals in Zweifel gezogen, da der um Rat befragte Schweizer Maler und Kunstwissenschaftler Johann Heinrich Meyer (1760-1832), seit 1791 Direktor der Freien Zeichenschule Weimar, eine Beteiligung Goethes an dem Werk ausschloss ${ }^{43}$. Lavater war daraufhin zunächst sehr verunsichert, vermutete dann aber, dass die Zeichnung von Tischbein stammen könne ${ }^{44}$. Kurz darauf erhielt er einen Brief von Meyer, in dem dieser das Bild als ein Gemeinschaftswerk von Johann Georg Schütz (1755-1815), Friedrich Bury (1763-1823) und Tischbein bezeichnete. Meyer sicherte Lavater jedoch zu, diese Erkenntnis für sich zu behalten ${ }^{45}$. Daraus erklärt sich gleichwohl, dass die von dem bekannten Zürcher Buchdrucker und Lithographen Joseph Brodtmann erstellten Abdrücke folgende Unterschrift tragen:

«Goethe in sinniger Betrachtung unter römischen Antiquitäten. Nach dem von Ihm Selbst gezeichneten Original, welches er in Italien einem Seiner begleitenden Freunde in den Jahren zwischen 1787-1788 zum Andenken gab.» ${ }^{46}$

Erst 1855 erfolgte eine Berichtigung durch Goethes letzten Sekretär Christian Schuchardt. In einer Mitteilung im «Weimarer Sonntags-Blatt» bezeichnete dieser allerdings Meyer als einen der Zeichner und schloss eine Beteiligung Tischbeins aus ${ }^{47}$. Obwohl auch Schuchardt seine Angaben von Meyer bezogen haben will, scheint doch dessen erste Version im Brief an Diethelm Lavater II glaubwürdiger, in dem er schreibt, dass «im Umriss W. Tischbeins Feder, in der Landschaft der G. Schütz aus Frankfurt zu erkennen und die Figur von F. Buri aus Hanau ausgetuscht» $\mathrm{se}^{48}$.

Ausgehend von der Tatsache, dass sowohl Meyer als auch Schütz und Bury zusammen mit Goethe im Hause Tischbeins in Rom als Gäste weilten, wird in heutigen Ausstellungskatalogen die Zeichnung als Gemeinschaftswerk Tischbeins, Schütz' und Burys angegeben ${ }^{49}$.

43 Vgl. Anm. 32.

44 Vgl. Anm. 26.

45 Brief von Johann Heinrich Meyer an Diethelm Lavater II vom 29. Juni 1832, FA Lavater 1781/14, Zentralbibliothek Zürich.

46 Vgl. dazu Anm. 35, S. 9.

47 Vgl. Anm. 3.

48 Vgl. Anm. 45.

49 Vgl. Anm. 27 und Boyle, N., Goethe. Der Dichter in seiner Zeit, Band I 1749-1790, München, 1995, 494f. 


\section{Diskussion}

Die Geschichte der Zeichnung «Goethe in der Campagna di Roma» zeigt eindrucksvoll die freundschaftlichen, aber auch die weitgespannten wissenschaftlichen Beziehungen der Familie Lavater. Ausgangspunkt dafür bildeten Kontakte zwischen Goethe, Tischbein und Johann K. Lavater in der zweiten Hälfte des 18. Jahrhunderts. Schon Diethelm Lavater I hatte durch seine Mitgliedschaft in der Freimaurerloge sowie die Korrespondenz mit Trommsdorff Verbindungen nach Deutschland aufgebaut, die durch den Aufenthalt Diethelm Lavaters II an Trommsdorffs Institut und sein Studium in Jena und Göttingen noch gefestigt wurden. Das hohe Ansehen Johann K. Lavaters in Deutschland dürfte dabei für beide hilfreich gewesen sein. Die Korrespondenz der Apotheker Lavater weist neben wissenschaftlichen Themen auch ein grosses Interesse an Politik und Kultur aus. Grundlage dafür war die natur- und geisteswissenschaftlich anspruchsvolle Bildung innerhalb der Familie Lavater und deren rege Teilnahme am gesellschaftlichen Leben. Diethelm Lavater II, wie sein Vater Diethelm I ein grosser Kunst- und Musikliebhaber, nutzte seine in der Zeit der wissenschaftlichen Ausbildung entstandenen Kontakte nach Deutschland für einen Kunsttransfer an den Weimarer Hof. Bezeichnend ist, dass für ihn nicht der finanzielle, sondern der ideelle Gewinn im Vordergrund stand. Somit unternahm das Bildnis Goethes eine ähnliche Reiseroute wie zuvor seine Schöpfer und der dargestellte Dichter - von Italien über die Schweiz nach Deutschland. 